\title{
Yuri L. Daletskii and the Development of Infinite Dimensional Analysis
}

Yana Belopolskaya (St Petersburg University of Architecture and Civil Engineering, Russia) and Alexei Daletskii (The University of York, UK)

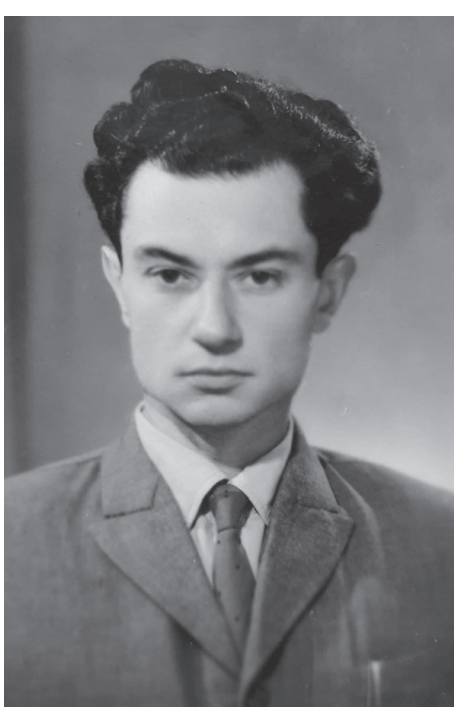

demobilisation from the army, 1946.
Yuri L. Daletskii after

Yuri L'vovich Daletskii was born on 16 December 1926 in Chernigov, a small town near Kiev in Ukraine, at that time a republic of the Soviet Union, to a family of mixed Jewish-Polish descent. The English spelling of Yuri's surname is ambiguous, other versions (according to MathSciNet) being Dalecky, Daleckii and Daletsky. Yuri was raised by his mother and stepfather. At the beginning of the 1930s the family moved to Kiev, where Yuri went to school. The Second World War did not allow him to finish it. Fleeing from advancing German Nazi troops, Yuri's family headed to Eastern Ukraine and then the Caucasus. Yuri was then sent further east to Kemerovo, a city in Western Siberia, where he lived with his uncle.

At an early age, Yuri fell in love with mathematics and music. He had inherited a good voice from his parents and sang in a children's choir (Yuri's cousin became a professional opera singer). At the age of thirteen, Yuri survived paralytic poliomyelitis. Although the illness destroyed his singing voice and any chance of a professional musical career, his love and deep understanding of music became an important part of his personality. In order to overcome the illness' residual effects, Yuri took up gymnastics and became quite proficient at it, staying physically strong and fit throughout his life.

In his teens, Yuri spent a lot of time reading mathematical books, and his mathematical knowledge far exceeded school level. In 1944 he was admitted as a student to the Mining Institute in Kemerovo. Soon after, he volunteered for the Soviet army and participated in the war against Japan.

Yuri was demobilised in 1946 and returned to Kiev, where he started his studies at the Mechanics and Mathematics Faculty of Kiev University. Alongside the study and research work, Yuri actively participated in students' organisations and the public life of the university. Just weeks before the final examinations, he was expelled for criticising the rector (although the formal reason given was the absence of a secondary school certificate). Dur- ing the next year, Yuri passed all school exams and was subsequently allowed to complete his university programme. He graduated in 1951, having written several research papers by that time. These works became the foundation of his $\mathrm{PhD}$ thesis completed under the supervision of Selim Krein, who played an important role in the development of the functional analysis school in Kiev. Soon after, Selim moved to Voronezh

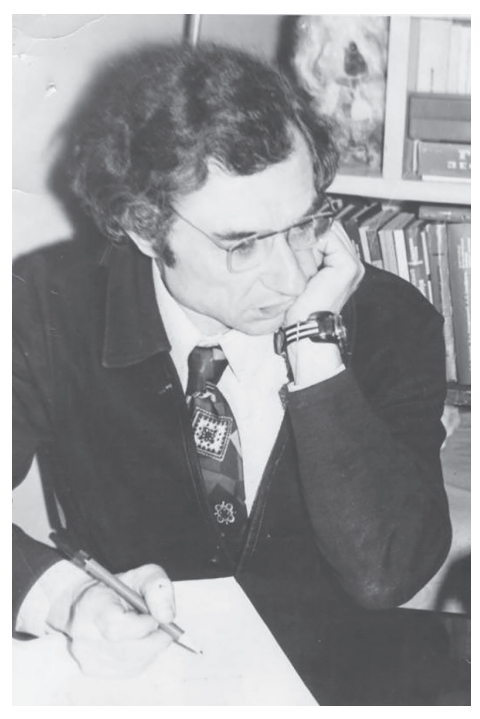

At work (1971). University. Yuri was very close to his teacher, always acknowledged his influence and remained in contact with him for his whole life.

In 1951, Yuri took up a position at the Kiev Polytechnic Institute (The National Technical University of Ukraine at present), where he would remain for the entirety of his career, first as an assistant and eventually as a full professor and member of the Ukrainian Academy of Sciences. Yuri played a major role in forming the mathematical curriculum of the Institute. In the 70s and $80 \mathrm{~s}$ he developed the mathematical programmes of the departments of Applied Mathematics, Mathematical Methods of System Analysis and the (new at that time) Faculty of Physics and Technology. Additionally, Yuri was one of the leaders of the successful independent post-graduate programme "Mathematics for Engineers", which was taught in Kiev for nearly two decades. Later, in the 90s, he also led mathematical programmes at the newly-founded Soros University.

Very soon Yuri became a significant figure on the Kiev mathematical scene. At that time, mathematical life in Soviet research centres was concentrated around big inter-institutional seminars, famous examples being Gelfand's and Dobrushin's seminars in Moscow. Yuri supervised major Kiev seminars "Random processes and distributions in functional spaces" (together with A. Skorokhod) and "Algebraic Structures in Mathematical Physics". He was also an important contributor to the seminar "Group methods in solid-state physics". Due to his friendly and energetic personality and vast knowledge of a variety of mathematical fields, Yuri played a 


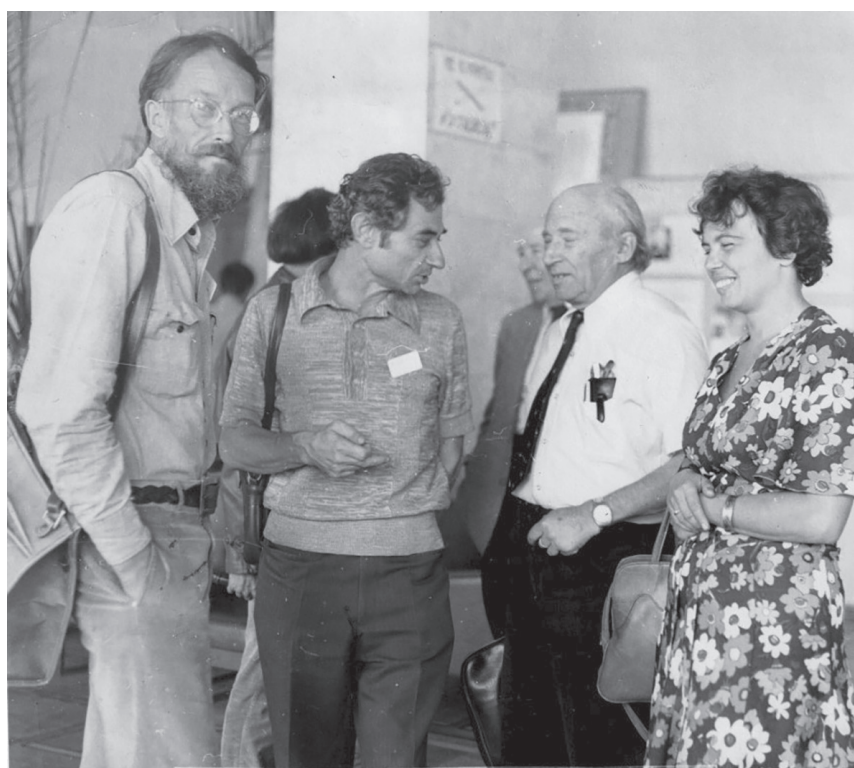

Symposium on soliton theory, Kiev, 1979. From left to right: H.P. McKean, Yu. L. Daletskii, I.M. Gelfand, Ya. I. Belopolskaya.

connecting role between different mathematical groups in Kiev. He shared Israel Gelfand's philosophical view of mathematics as a single interconnected field, and was always interested in invariant algebraic structures "hiding" in various mathematical theories. To a large extent that was the philosophy and motivation of his seminar "Algebraic Structures in Mathematical Physics", which had a truly interdisciplinary flavour on the interface of algebra, geometry, stochastic analysis and mathematical physics.

Yuri became well known within the mathematical world of the Soviet Union, which was, however, rather isolated from the rest of the world. He was an active participant and contributor to many conferences, among them the famous Voronezh Winter Mathematical School organised by Selim Krein, which year after year attracted the mathematical elite of the country.

Yuri's development as a scientist was very much influenced by his collaboration with two mathematical

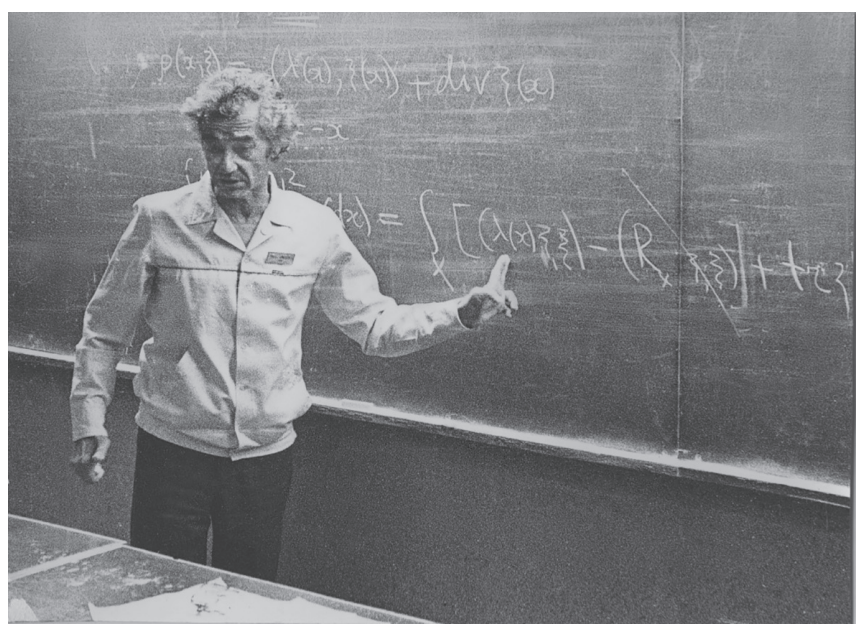

Fifth International Vilnius Conference on Probability Theory and Mathematical Statistics, Vilnius 1989.

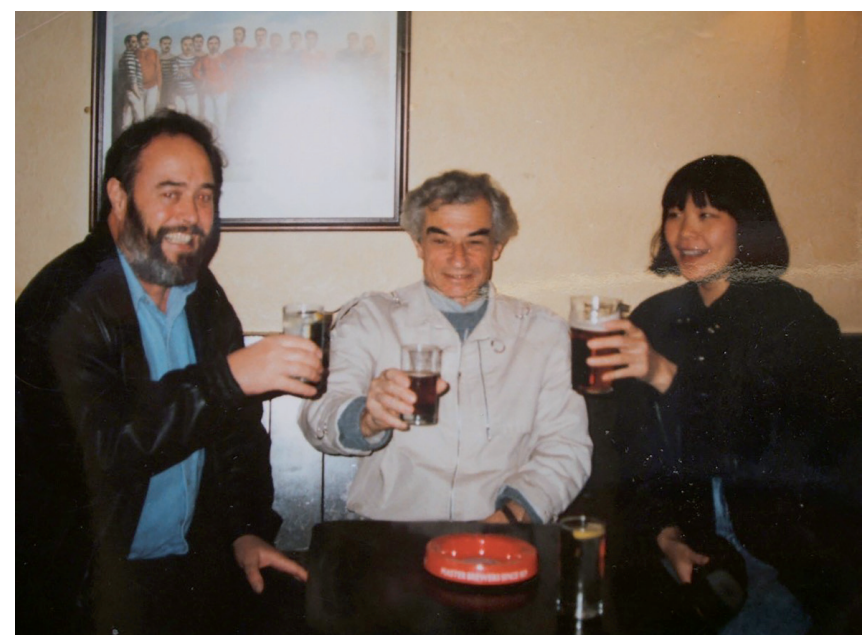

Warwick, 1991. From left to right: K.D. Elworthy, Yu.L. Daletskii, X.-M. Li.

giants of the 20th Century - Mark Krein and Israel Gelfand. Mark Krein lived in Odessa, and Yuri liked to rent a holiday house by the sea, staying there with his family and combining work with long offshore swims. This collaboration resulted in the joint book Stability of solutions of differential equations in Banach spaces (1974), where the infinite-dimensional version of the Lyapunov stability theory was developed.

Yuri had been a frequent participant of Gelfand's seminar at the Moscow State University. More direct collaboration started, however, in the early 80s, when Gelfand was spending summers at his wife's home in Kiev. This collaboration lead to a number of works on non-commutative differential geometry and topology (joint with B. Tsygan, L. Takhtajan and others).

Yuri was a true pioneer of global infinite-dimensional stochastic analysis. His research in this field, rooted in Gelfand's ideas, formed the contents of two books: Measures and Differential Equations in Infinite-Dimensional Spaces (jointly with S. Fomin and actually finalised by Yuri after Fomin's death in 1983; the English translation appeared in 1991), and Stochastic Equations and Differential Geometry (jointly with Ya. Belopolskaya, 1989; English translation 1990). This research was acknowledged around the world, and soon after 1991 Yuri became a frequent traveller and visited most of the world's important mathematical research centres.

In the 1950s, a strong motivation for the development of the analysis of measures and random processes in infinitely many dimensions was the need for a rigorous theory of Feynman integrals. After Mark Kac's results on parabolic equations and his derivation of the celebrated Feynman-Kac formula for the Wiener measure, it looked possible to implement a similar approach in the study of the Schrödinger equation and represent its solutions via integrals with respect to probability measures on path spaces. Yuri showed (independently of R. Cameron) that no such measures could be constructed, that is, they appeared to be quasi-measures of unbounded variation. He developed a rigorous construction of the integrals with respect to such quasi-measures, including abstract 
Gaussian quasi-measures with complex variance parameters.

As a technical tool for these studies, Yuri proved a chronological product formula for evolution families of operators. In the simplest case, for a generator $A$ of a strongly continuous semigroup $e^{t A}$ and a bounded operator $B$ in a Banach space, the semigroup generated by the sum $A+B$ has the following form:

$$
e^{t(A+B)}=\lim _{\Delta t_{k} \rightarrow 0} \prod_{k} e^{\Delta t_{k} A} e^{\Delta t_{k} B}, \text { where } \sum_{k} \Delta t_{k}=t .
$$

This result, usually called the Trotter product formula in the literature, was obtained by Yuri independently and extended to the case of evolution families generated by non-autonomous equations $\dot{\phi}(t)=A(t) \phi(t)$ and $\dot{\psi}(t)=B(t) \psi(t)$. It lead to the notion of operator-valued multiplicative integrals, and was in turn used by Yuri to prove Feynman-Kac type formulae for abstract evolution equations, including various equations of parabolic, hyperbolic and Schrödinger type. This, in particular, allowed him to give a rigorous derivation of the Feynman-Kac formula for Schrödinger type equations with bounded smooth potentials; a generalisation for singular potentials was later obtained by Nelson.

Furthermore, Yuri went on to consider abstract evolution equations in infinite-dimensional (Hilbert or Banach) spaces. Simultaneously with Leonard Gross, he defined the infinite-dimensional Laplacian for functions

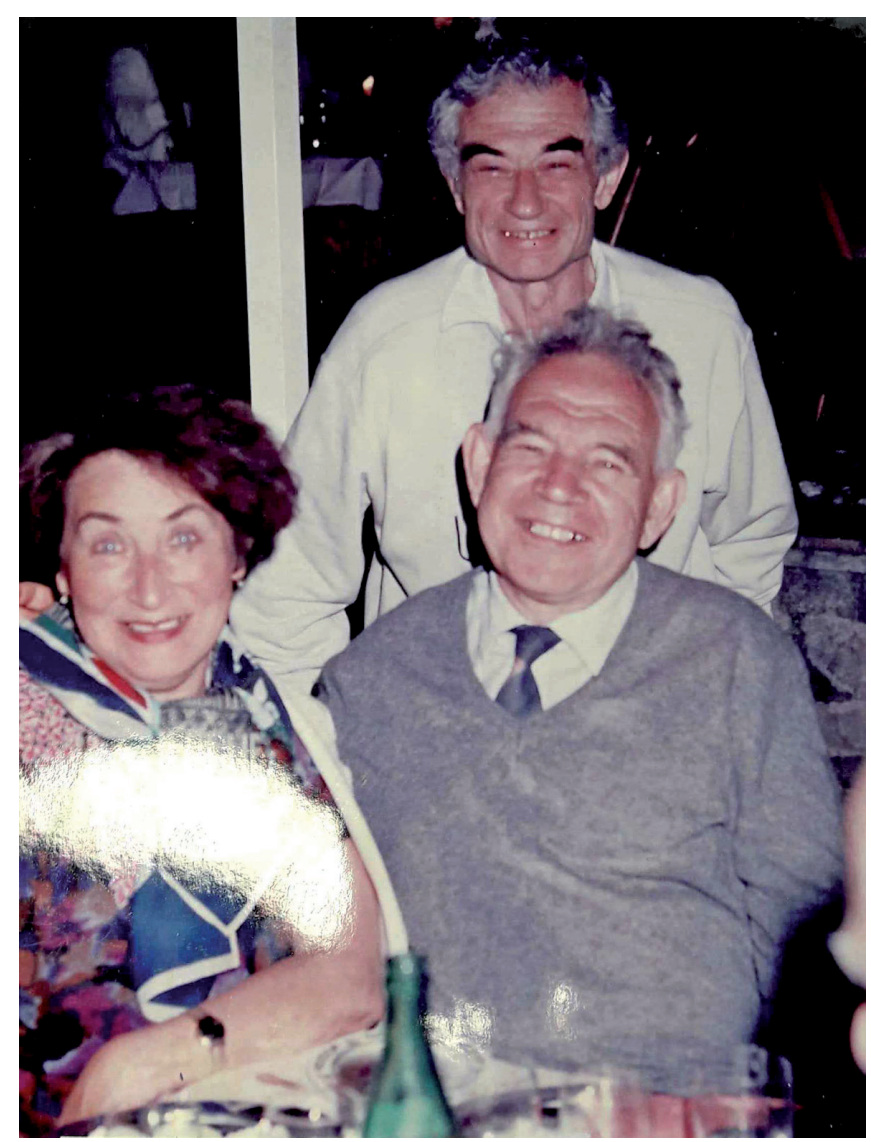

Capri, 1993. From left to right: L.P. Daletskaya, Yu.L. Daletskii, R.A. Minlos.

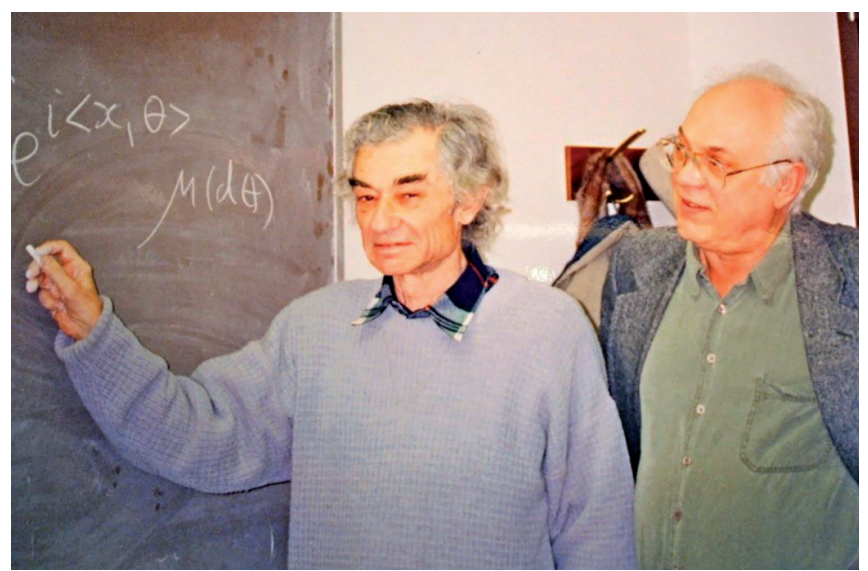

Michigan, 1996. From left to right: Yu. L. Daletskii, A. V. Skorokhod.

with trace-class second derivatives. L. Gross (J. Funct. Anal. 1967) refers to Yuri's paper "Differential equations with functional derivatives and stochastic equations for generalised random processes" (Dokl. Akad. Nauk USSR 1966, with yet another spelling of Yuri's surname as Daletzkii), which had previously been unknown to him. These ideas led Yuri to the development of the general theory of stochastic differential equations in Banach spaces and corresponding probabilistic representations of solutions of parabolic equations, in the framework of Gelfand triples, building upon the classical finite-dimensional results of Itô, Gikhman and Skorokhod. This work formed a foundation for a variety of theories, including those of stochastic partial differential equations, stochastic quantisation of infinite particle systems and smooth measures on infinite-dimensional spaces.

Yuri's research was much concerned with differential-geometric aspects of global stochastic analysis on infinite-dimensional spaces. In a series of joint works with Ya. Belopolskaya, a concept of a stochastic differential equation on a Banach manifold endowed with a Hilbert-Schmidt structure was developed. The Itô stochastic differential was considered as a section of a special vector bundle (the Itô bundle), highlighting its geometric nature. This research was initiated in the paper "Diffusion processes in smooth Banach spaces and manifolds" (Trans. Moscow Math. Soc.,1978), and culminated in their book Stochastic Equations and Differential Geometry, which also covered the case of stochastic equations associated with quasi-linear parabolic equations on Banach spaces and manifolds.

Other important contributions to the development of infinite-dimensional analysis were the theory of smooth measures on Banach manifolds and its connection with the notion of the Hitsuda-Skorokhod integral, the theory of Lévy-Laplacian, the theory of bi-orthogonal expansions and non-Gaussian distributions (with S. Albeverio, Yu. Kondratiev and L. Streit), to name but a few.

A lot of Yuri's work was done in collaboration with his students and colleagues. He successfully supervised $30 \mathrm{PhD}$ students, some of whom became renowned scientists. A great lecturer and passionate teacher, he liked 
Albert Einstein's quote "Student is not a container you have to fill but a torch you have to light up." He was always generous in sharing his ideas and supporting his students, collaborators and colleagues, and brave enough to stand up for them if needed. Always full of energy, with deep knowledge of history, literature and poetry, he liked being surrounded by people and felt comfortable in any company. His other favorite quote was that of the famous Soviet biologist Timofeev-Ressovsky: "Science is a jolly lady and does not tolerate spiderish seriousness."

Yuri passed away in December 1997, a couple of days before his 71st birthday. How is he remembered? The two decades after his departure have given us the answer: not only as a brilliant scientist and teacher, but also as a bright and cheerful man, loved by his family, friends and colleagues, who had lived his life to the full.

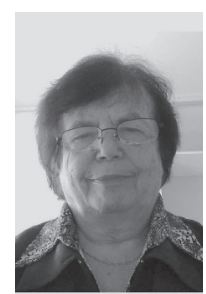

Yana Belopolskaya [yana@yb1569.spb. edu] is a Professor of Mathematics at the St.Petersburg State University of Architecture and Civil Engineering. She graduated from the Kiev Polytechnic Institute, then got her PhD in the Kiev Institute of Mathematics (1965) and her Doctor of Science degree in the Leningrad Department of the Steklov Mathematical Institute (1990). She works on stochastic differential equation theory, nonlinear PDE and systems theory and connections between these two theories.

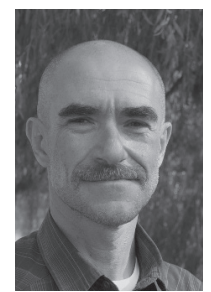

Alexei Daletskii [alex.daletskii@york. ac.ukJ obtained his PhD and Doctor of Science degrees from the Institute of Mathematics NAS, Kiev, Ukraine. He has held full-time positions in Bonn and Nottingham and works at present at the Mathematics Department of the University of York, UK. His main research interests are in stochastic analysis and its applications in mathematical physics. Alexei is Yuri Daletskii's son. 